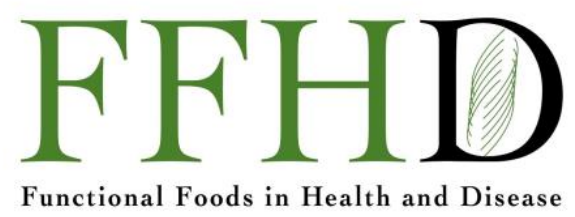

\title{
Effect of combining sleep-promoting food intake and electric field application on sleep in healthy participants: A pilot study
}

\author{
Takaki Nedachi $^{1}$, Kaoru Haketa ${ }^{1}$, Shinji Harakawa ${ }^{1,2}$, Naoki Miura ${ }^{3}$, Koji Wakame ${ }^{4 *}$ \\ ${ }^{1}$ Research and Development Department, Hakuju Institute for Health Science, Tokyo, 151-0063, Japan; 'Bio-Self-Regulating \\ Science Laboratory, Obihiro University of Agriculture and Veterinary Medicine, Obihiro, Hokkaido, 080-8555, Japan; ${ }^{3}$ Miura \\ Clinic, Medical Corporation Kanonkai, Kita-ku, Osaka, 530-0044, Japan; ${ }^{4}$ Faculty of Pharmaceutical Sciences, Hokkaido \\ University of Science, Sapporo, Hokkaido, 006-8585, Japan
}

*Corresponding Author: Koji Wakame PhD, Faculty of Pharmaceutical Sciences, Hokkaido University of Science, Sapporo, Hokkaido 006-8590, Japan

Submission Date: November $3^{\text {rd }}, 2021$; Acceptance Date: November 29th 2021 ; Publication Date: December $16^{\text {th }}, 2021$

Please cite this article as: Nedachi T., Haketa K., Harakawa S., Miura N., Wakame K. Effect of combining sleep-promoting food intake and electric field application on sleep in healthy participants: A pilot study. Functional Foods in Health and Disease 2021; 11(12): 659-672. DOI: https://www.doi.org/10.31989/ffhd.v11i12.861

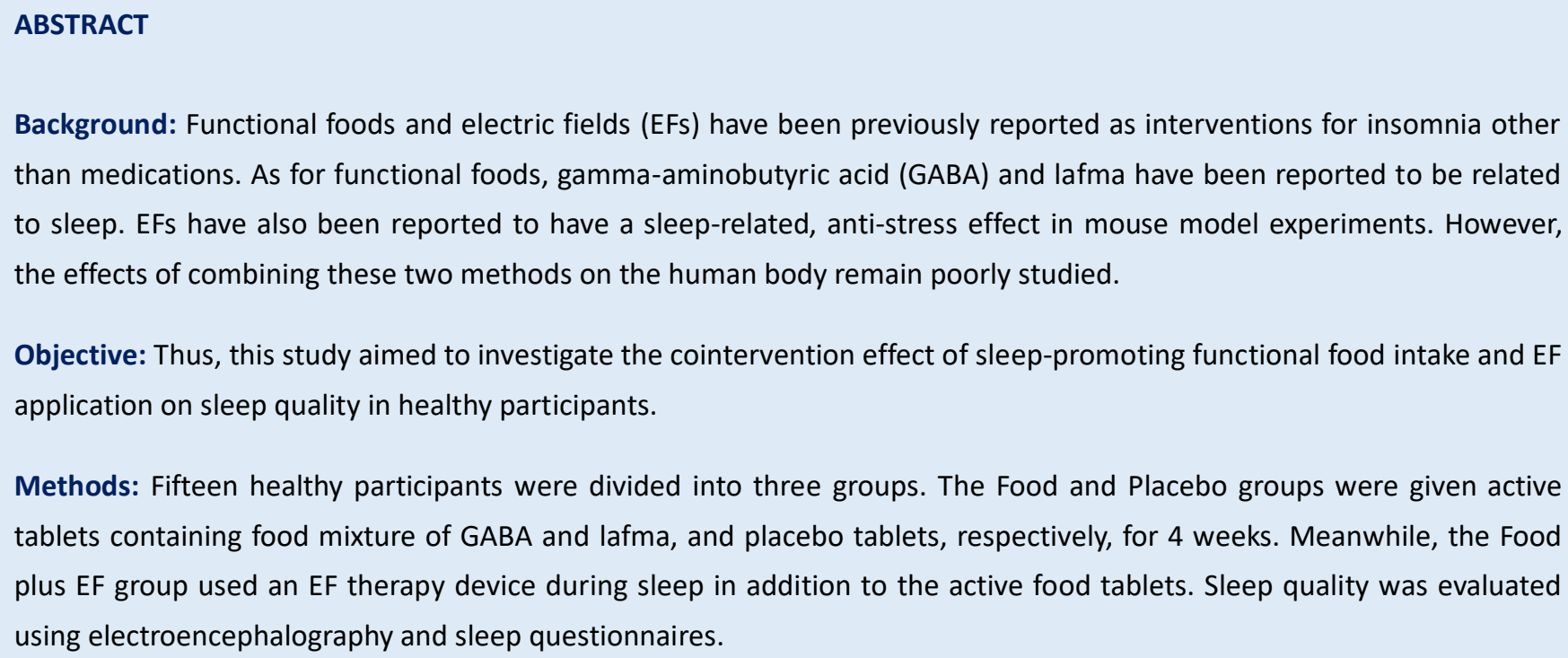
than medications. As for functional foods, gamma-aminobutyric acid (GABA) and lafma have been reported to be related to sleep. EFs have also been reported to have a sleep-related, anti-stress effect in mouse model experiments. However, the effects of combining these two methods on the human body remain poorly studied.

Objective: Thus, this study aimed to investigate the cointervention effect of sleep-promoting functional food intake and EF application on sleep quality in healthy participants.

Methods: Fifteen healthy participants were divided into three groups. The Food and Placebo groups were given active tablets containing food mixture of GABA and lafma, and placebo tablets, respectively, for 4 weeks. Meanwhile, the Food plus EF group used an EF therapy device during sleep in addition to the active food tablets. Sleep quality was evaluated using electroencephalography and sleep questionnaires. 
Results: Sleep efficiency (SE) was significantly higher in the Food group and the Food plus EF group than the Placebo group at 4 weeks. The Food plus EF group also had a significantly higher SE involving sleep latency.

Conclusions: Food mixture containing known sleep-promoting ingredients such as GABA and lafma can improve sleep quality, and the improvement effect can be enhanced when administered in combination with an EF.

Keywords: electric field therapy, Kumasasa (Sasa senanensis), electric fields, sleep quality, electroencephalography

Clinical trial registration: Approval No.: R1812; Approval date: 21 Feb. 2019

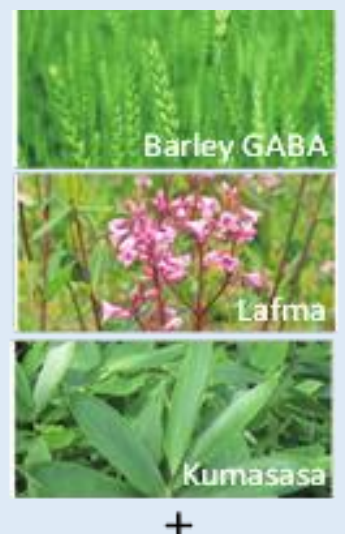

$+$

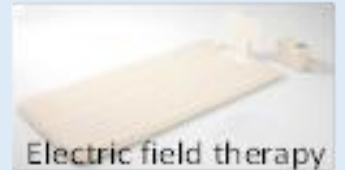

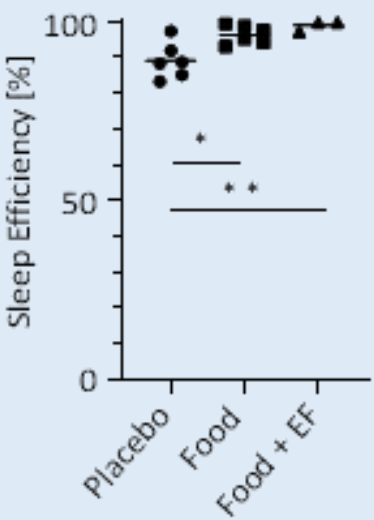

Sleep efficiency is improved by consuming food containing sleep-related ingredients such as GABA and lafma, and is enhanced when the administration of such food formula is combined with Electric field (EF) application.

(CFFC 2021. This is an Open Access article distributed under the terms of the Creative Commons Attribution 4.0 License (http://creativecommons.org/licenses/by/4.0)

\section{INTRODUCTION}

In today's urban living, mental health problems and reduced sleep quality are becoming a problem because of changes in the environment and various lifestyles. For example, an epidemiological study involving Chinese university students revealed problematic mobile phone use is associated with mental health and reduced sleep quality [1]. A Japanese study also reported that sleep duration depends on seasonal changes; in other words, sleep duration is longest in the winter and shortest in the summer [2]. This relationship between season and sleep is more prevalent in middle-aged people than in younger people [3]. In addition, a diet high in carbohydrates, such as sweets and noodles, and low in vegetables was associated with a lower sleep quality among Japanese female workers. In elderly population living in urban areas, dietary content and appetite stimulation are important to maintain sleep quality. Thus, sleep quality varies depending on lifestyle, age group, season, and dietary habits [4].

Given these health problems experienced by modern people, the Ministry of Health, Labor, and Welfare has approved the use of electric field (EF) therapy devices for insomnia [7]. This type of device treats the human body with EFs at an extremely low frequency to modulate the blood flow, nervous system, and endocrine system, for prevention and treatment of 
various symptoms such as insomnia, headache, shoulder stiffness, and chronic constipation. For example, animal experiments showed that EFs could suppress stress hormones in immobilization-induced mice; hence, EFs have an anti-stress effect, considering that the EF effects depend on the strength, duration, and area of EF exposure [5-6]. Clinically, EF improves sleep [7] and increases the electroencephalographic (EEG) theta wave during daytime [8]. The effects of EF on insomnia can be explained by the anti-stress effect.

Meanwhile, functional food ingredients for sleep such as glycine [9], L-serine [10], L-theanine [11], ornithine [12], gamma-aminobutyric acid (GABA) [13], lactoferrin [14], vitamin B [15], asparagus [16], and lafma [17], are related to sleep and relaxation. These ingredients are grouped into amino acids and their analogs, herbal extracts, and probiotics, which all reportedly enhance sleep quality and recovery from fatigue. Some of the previous reports suggested improvement in sleep was associated with stress-relieving effect [12-13, 16].

Although solitary administration of EFs or functional foods has been reported to improve health problems and sleep quality in humans, the effectiveness is not enough. Moreover, the effect of combining these two approaches on sleep remains unreported. Thus, this study aimed to investigate the cointervention effect of a food mixture containing sleep-enhancing ingredients and EF on sleep.

\section{METHODS}

A randomized, double-blind, placebo-controlled, parallel intergroup comparison study was conducted as a human trial. The investigational review board of Miura Clinic approved this study (Approval No.: R1812; Approval date: 21 Feb. 2019)

(UMIN Trial ID: UMIN000035977)

Participants: Healthy middle-aged females were recruited.

A. The inclusion criteria were: a. healthy females over $40-64$ years of age

b. after menopause

c. with lower sleep quality

d. without sleep medication

e. well-informed about the aim and content of the study, competent to consent, voluntarily applied for participation, and gave a written consent.

B. The exclusion criteria were:

a. volunteers with medical history of liver, kidney, digestive system, heart, and/or any other disease which can interfere the study results

b. diagnosed with heart disease limiting strenuous exercise

c. with implantable medical electronic devices vulnerable to electromagnetic interference

d. with wearable medical electronic devices (ex. electrocardiographs, pacemakers)

e. with abnormal liver and kidney function tests

f. under treatment

g. with food and/or drug allergies

h. playing hard sports and/or on a diet

i. unable to refrain from taking health food and/or quasi-drug

j. taking continuously over-the-counter drug

k. taking health food and/or medication for sleep improvement

I. using sleep-promoting bedding and/or devices

$m$. being or having been a user of EF therapy devices

n. drinking excessive alcohol or being unable to refrain from drinking during the EEG measurement and medical checkups

o. during pregnancy or having a plan to be pregnant and breastfeeding

p. currently participating in, or having a plan to participate in another clinical study

q. being judged inappropriate by the attending doctor 
Test samples and the administration method: The ingredients of the three tablets for our food sample are listed in Table 1. Main functional ingredients for sleep were lactic-fermented barley GABA (120 mg), lafma extract $(50 \mathrm{mg}), 0.1 \%$ vitamin B12 (3 $\mathrm{mg})$, and vitamin B6 (1.5 mg) (Table 1). For the placebo sample, the main ingredients of the three tablets were dexitrin (264 mg) and maltitol (264 mg). Of note, the placebo tablets resembled the food sample in appearance.

In both samples, one package (containing 3 tablets) was administered daily, orally with water, 30-60 minutes before sleep, with no chewing, for 4 weeks.

Table 1. Ingredient list of the food samples.

\begin{tabular}{|l|l|l|}
\hline Ingredients per 3 tablets & Investigational food & Placebo food \\
\hline Lactic-fermented barley GABA & $120.0 \mathrm{mg}$ & - \\
\hline Lafma extract & $50.0 \mathrm{mg}$ & - \\
\hline Vitamin B12 (0.1\%) & $3.0 \mathrm{mg}$ & - \\
\hline Vitamin B6 & $1.5 \mathrm{mg}$ & - \\
\hline Kumasasa powder & $50.0 \mathrm{mg}$ & - \\
\hline Dexitrin & - & $264.0 \mathrm{mg}$ \\
\hline Maltitol & - & $264.0 \mathrm{mg}$ \\
\hline Cellulose & $400.0 \mathrm{mg}$ & $112.2 \mathrm{mg}$ \\
\hline Granular silicon oxide & $13.0 \mathrm{mg}$ & $6.6 \mathrm{mg}$ \\
\hline Calcium stearate & $22.5 \mathrm{mg}$ & $13.2 \mathrm{mg}$ \\
\hline Methyl cellulose & $30.0 \mathrm{mg}$ & $30.0 \mathrm{mg}$ \\
\hline HPMC & & \\
\hline Titanium oxide & & $690.0 \mathrm{mg}$ \\
\hline Glycerine & & \\
\hline Total & $690.0 \mathrm{mg}$ & \\
\hline
\end{tabular}

GABA, gamma-aminobutyric acid; HPMC, hydroxypropyl methylcellulose.

EF exposure system: We used an EF therapy device for home use (Healthtron HEF-N6000WG; Approval No.: 228AKBZX00085000) (Fig. 1). Antiphase voltages up to $5000 \mathrm{~V}$ were applied to the upper and lower electrodes, causing $60 \mathrm{~Hz}$, sinusoidal EF generation between the electrodes. While sleeping up to 8 hours, participants were treated with the EFs on the pad.

\section{Upper electrode}

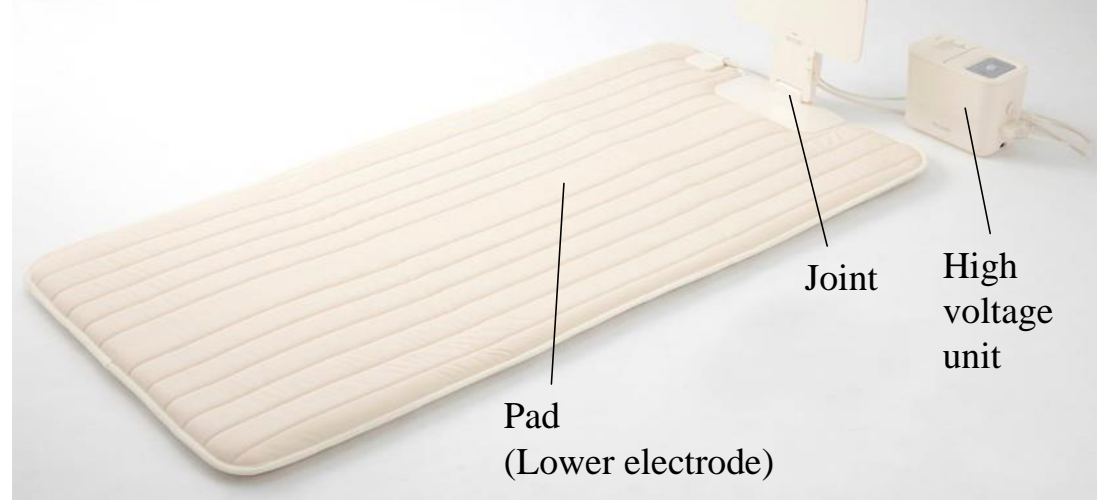

Figure 1. EF therapy device. Antiphase voltages were applied to the upper and lower electrodes, leading to sinusoidal EF generation between the electrodes. Participants were treated with the EFs during sleep on the pad. EF, electric field. 


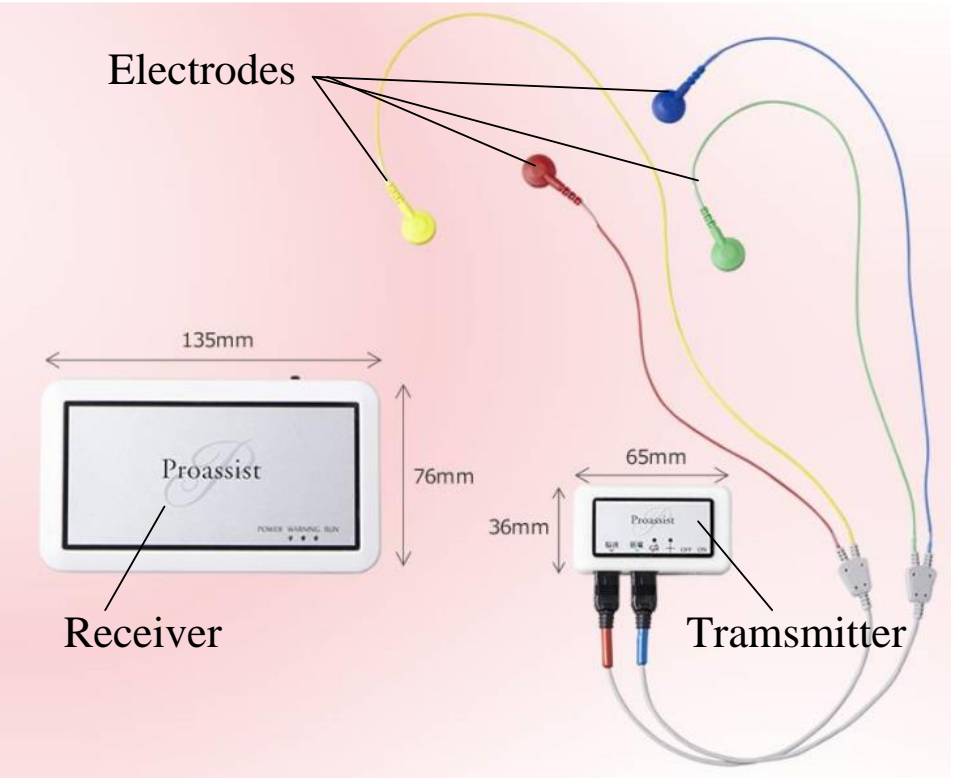

Figure 2. Sleep measuring device: "Brain wave sensor ZA-X (Ten)." Electroencephalogram and electromyogram were derived from the electrodes. Signals were wirelessly transmitted and received for sleep analysis.

Sleep measurement device and sleep parameters: Sleep

EEG was measured by the EEG sensor ZA-X (Proassist, Ltd., Osaka, Japan) (Fig. 2).

The changes in the stages of sleep during night are represented as hypnograms (Fig. 3). Rapid eye movement (REM) and non-REM sleep alternate in the sleep cycle during night. Sleep is classified in four stages, non-REM sleep stage N1, N2, N3, and REM sleep stage. Non-REM sleep becomes deeper from N1 to N3.

Sleep parameters were defined as below.

Sleep Period Time (SPT) [min]: The duration of time from sleep onset to final awakening. Sleep Total Sleep
Time (TST) [min]: The actual sleep time except for intermittent awakening in SPT.

Sleep Efficiency (SE) [\%]: (TST/SPT) × 100 .

Sleep Latency (SL) [min]: The duration of time from bedtime to the onset of sleep.

SE including SL [\%]: (TST/(SPT + SL)) × 100.

Stage N3: Twenty percent or more of an epoch consists of slow wave activity, or EEG delta wave of 0.5 $\mathrm{Hz}$ to $2 \mathrm{~Hz}$ with peak-to-peak amplitude of more than 75 $\mu \mathrm{V}$.

Delta power: The power of EEG delta wave expressed in $\mu V^{2}$.

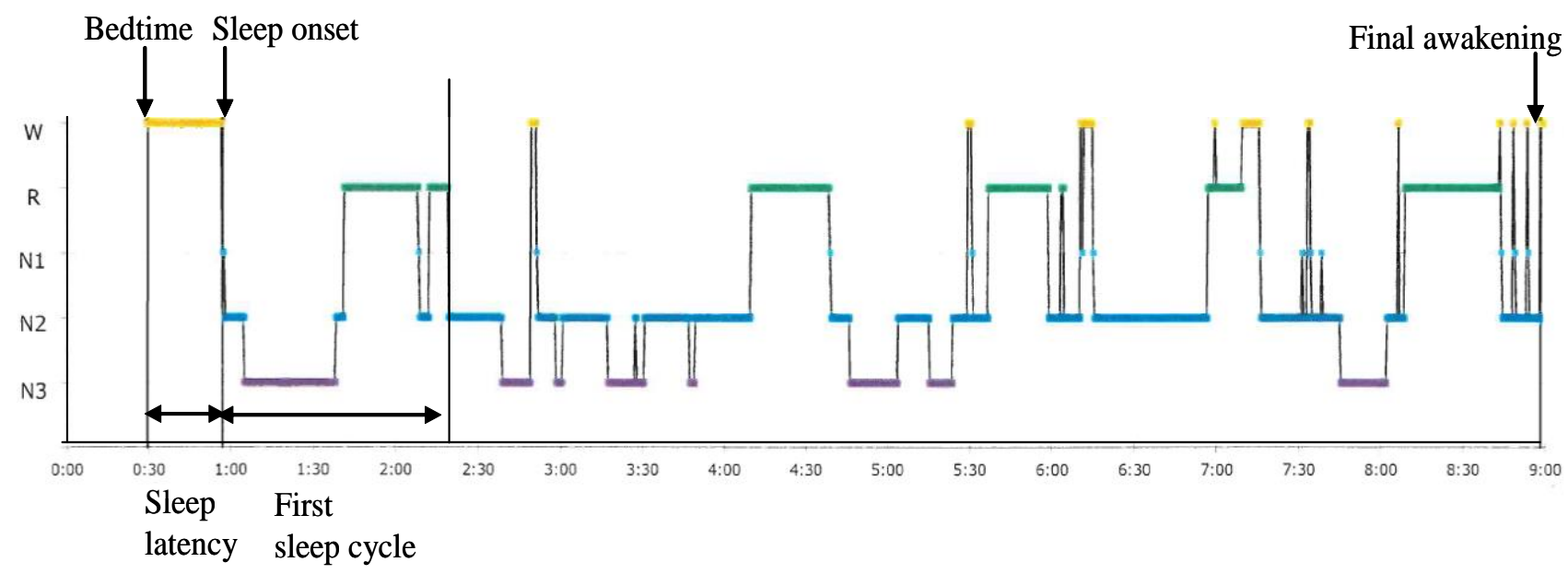

Figure 3. Hypnogram. Rapid eye movement (REM) and non-REM sleep alternate in the sleep cycle during night. Sleep is classified in four stages, non-REM sleep stage N1, N2, N3, and REM sleep stage. Non-REM sleep becomes deeper from N1 to N3. W, stage wake; R, stage REM; N1, non-REM sleep stage N1; N2, non-REM sleep stage N2; N3, non-REM sleep stage N3. 
Study design: Healthy females who fulfilled the inclusion criteria were selected and assigned to the following groups: Placebo group, administered with the placebo food; Food group, administered with the investigational food; and Food plus EF group, cotreated with the investigational food and the EF therapy device.

Study schedules and design are indicated in Fig. 4.
All participants visited the medical site and underwent intake and inquiry, physical tests, and questionnaire survey. After the measurement method was explained, they were offered investigational or placebo food and the EEG sensor was applied. Investigational devices were then sent to participants' home.

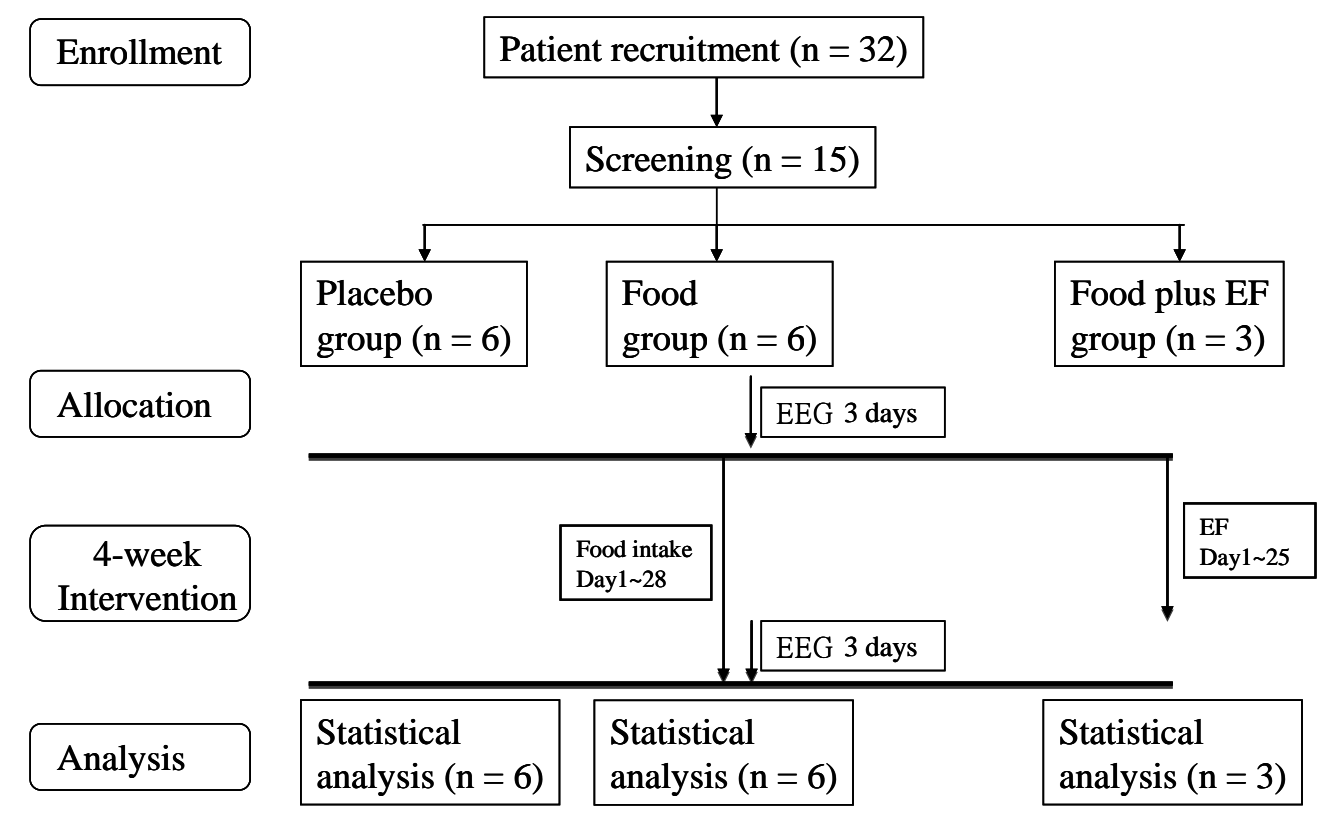

Figure 4. Flowchart of the trial. Out of 32 potential candidates recruited, 15 were selected after screening, of which 6 , 6, and 3 were assigned to the Placebo, Food, and Food plus EF groups, respectively. All participants were eligible.

For the Placebo and Food groups, sleep EEG was measured for 3 days before intake according to the user's manual at participants' home. Similarly, sleep EEG was measured for 3 days before the checkup at week 4 . Study food was administered from day 1 to day 28 .

For the Food plus EF group, the investigational device was used every night at bedtime according to the user's manual from day 1 to day 25 , whereas the investigational food was administered from day 1 to day 28. During the sleep EEG measurement period, the investigational device was not applied to prevent electric interference.

From day 1 to day 28, the participants were asked to record their placebo or investigational food intake, alcohol intake, and exercise in a sleep diary. They were also asked to record their activity during the sleep EEG measurement period. When they could not undergo checkups during the designated time, they were allowed to change the schedule before or after a 7-day period.

Subsequently, the participants were asked to revisit the site immediately after the 4-week intervention to conduct the week 4 (day 28) checkups, physical tests, and questionnaires.

Physical examination was conducted before and after the 4-week period. Likewise, sleep quality was evaluated using the Oguri-Shirakawa-Azumi (OSA) sleep inventory and visual analog scale (VAS) questionnaire for sleep and fatigue before and after the intervention. In addition, sleep EEG was measured by the EEG sensor ZA-X (Proassist, Ltd., Osaka, Japan) (Fig. 2). VAS questionnaire for physical condition was applied to the Food plus EF group only. 
Statistical analysis: Intergroup differences were evaluated at weeks 0 and 4 , where we used the Tukey-Kramer test for the sleep EEG and OSA sleep inventory and the Steel-Dwass test for the VAS questionnaire. Conversely, intragroup differences from week 0 to 4 were evaluated by paired sample $t$-test for clinical characteristics, sleep EEG, and OSA sleep inventory, and Wilcoxon signed rank test for the VAS questionnaire. We considered $p<0.05$ to be statistically significant.

\section{RESULTS}

Table 2 summarizes the participants' physical examination data. The Placebo, Food, and Food plus EF groups consisted of 6,6 , and 3 eligible participants, with a mean age of $55.7 \pm 4.9,55.8 \pm 4.2$, and $54.7 \pm 5.8$ years, respectively, indicating no statistical difference (Table 2). Other data also showed no statistical difference, except the reduced systolic blood pressure in the Placebo group after the 4-week intervention (Table 2).

Table 2. Physical characteristics of the participants.

\begin{tabular}{|c|c|c|c|c|c|c|c|c|c|}
\hline \multirow[b]{2}{*}{ Age( years) } & \multirow{2}{*}{$\begin{array}{l}\text { Group } \\
\text { Placebo }\end{array}$} & \multirow{2}{*}{$\frac{n}{6}$} & \multicolumn{3}{|l|}{0 week } & \multicolumn{3}{|c|}{4 week } & \multirow{2}{*}{$\begin{array}{l}p \text { value } \\
\text { - }\end{array}$} \\
\hline & & & 55.7 & \pm & 4.9 & & - & & \\
\hline & Food & 6 & 55.8 & \pm & 4.2 & & - & & - \\
\hline & Food $+E F$ & 3 & 54.7 & \pm & 5.8 & & - & & - \\
\hline \multirow[t]{3}{*}{ Height (cm) } & Placebo & 6 & 156.52 & \pm & 3.49 & & - & & - \\
\hline & Food & 6 & 160.98 & \pm & 5.42 & & - & & - \\
\hline & Food $+E F$ & 3 & 160.17 & \pm & 1.02 & & - & & - \\
\hline \multirow[t]{3}{*}{ Weight (kg) } & Placebo & 6 & 55.13 & \pm & 5.20 & 54.85 & \pm & 6.71 & 0.7036 \\
\hline & Food & 6 & 55.13 & \pm & 5.70 & 54.22 & \pm & 5.36 & 0.1331 \\
\hline & Food $+E F$ & 3 & 49.50 & \pm & 4.19 & 50.07 & \pm & 3.41 & 0.4145 \\
\hline \multirow[t]{3}{*}{ BMI (kg/m2) } & Placebo & 6 & 22.55 & \pm & 2.57 & 22.45 & \pm & 3.20 & 0.7322 \\
\hline & Food & 6 & 21.28 & \pm & 2.05 & 20.91 & \pm & 1.65 & 0.1402 \\
\hline & Food $+E F$ & 3 & 19.29 & \pm & 1.46 & 19.51 & \pm & 1.19 & 0.4137 \\
\hline \multirow[t]{3}{*}{ Systric BP (mmHg) } & Placebo & 6 & 123.7 & \pm & 18.5 & 118.7 & \pm & 19.3 & $0.0449 *$ \\
\hline & Food & 6 & 112.8 & \pm & 14.4 & 113.5 & \pm & 11.6 & 0.7799 \\
\hline & Food $+E F$ & 3 & 115.0 & \pm & 10.4 & 108.3 & \pm & 6.0 & 0.4532 \\
\hline \multirow[t]{3}{*}{ Diastric BP (mmHg) } & Placebo & 6 & 79.0 & \pm & 13.5 & 76.3 & \pm & 13.2 & 0.4600 \\
\hline & Food & 6 & 70.3 & \pm & 15.0 & 71.7 & \pm & 10.2 & 0.5880 \\
\hline & Food $+E F$ & 3 & 62.7 & \pm & 1.5 & 59.7 & \pm & 9.8 & 0.6440 \\
\hline \multirow[t]{3}{*}{ Pulse (/min) } & Placebo & 6 & 85.2 & \pm & 7.7 & 82.7 & \pm & 3.7 & 0.5177 \\
\hline & Food & 6 & 74.7 & \pm & 13.8 & 80.3 & \pm & 14.2 & 0.0848 \\
\hline & Food $+E F$ & 3 & 75.3 & \pm & 10.1 & 74.7 & \pm & 7.6 & 0.9534 \\
\hline
\end{tabular}

Intragroup changes were analyzed using paired sample $t$-test. $*$ : $p<0.05 \mathrm{BMI}$, body mass index; BP, blood pressure.

The food intake rate was $100.0 \% \pm 0.0 \%, 99.4 \% \pm$ $1.5 \%$, and $99.0 \% \pm 1.8 \%$ in the Placebo-, Food-, and Food plus EF group, respectively, with no statistical difference.

At 4 weeks, sleep efficiency (SE) was significantly higher in the Food group and the Food plus EF group than the Placebo group (Fig. 5); when sleep latency was involved in the SE, the Food plus EF group showed a significantly higher value than the Placebo group (Fig. 6).
Sleep latency and the amount of stage N3 were not significantly different between the three groups at week 4 (Fig. 7).

In contrast, power spectrum analysis of EEG revealed that the EEG delta power in the first sleep cycle was significantly higher in the Food plus EF group than in the Placebo group at week 4 (Fig. 8). 


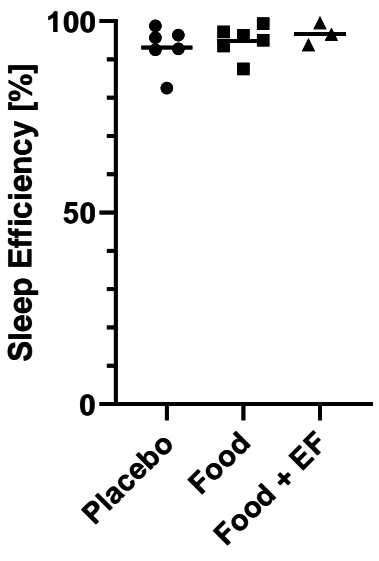

(a) 0 week

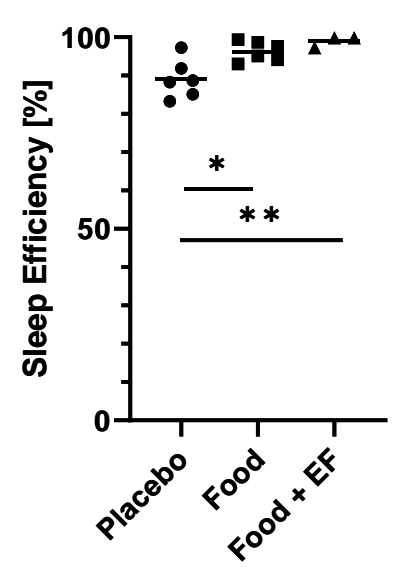

(b) 4 week

Figure 5. Sleep efficiency at weeks (a) 0 and (b) 4. Intergroup differences were analyzed using the Tukey-Kramer test. Sleep efficiency was significantly higher in the Food group and Food plus EF group than in the Placebo group at 4 weeks. ${ }^{* *}: p<0.01, *: p<0.05$. EF, electric field.

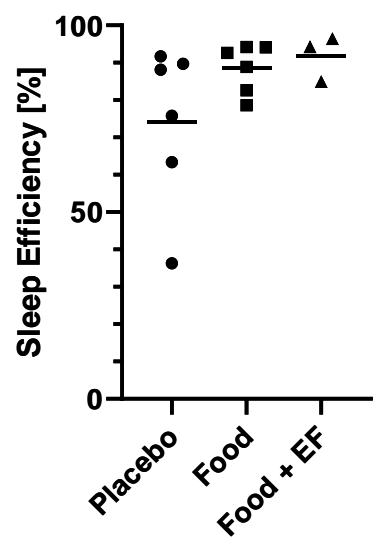

(a) 0 week

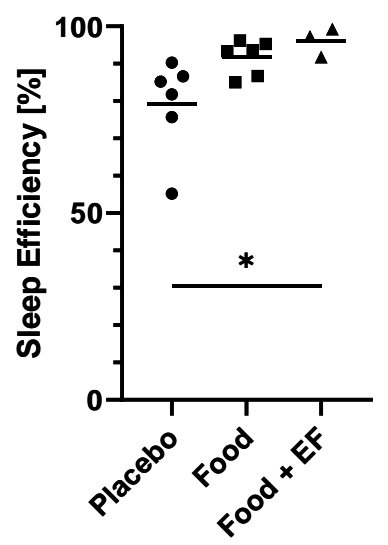

(b) 4 week

Figure 6. Sleep efficiency including sleep latency at weeks (a) 0 and (b) 4. Intergroup differences were analyzed using the Tukey-Kramer test. The Food plus EF group showed a significantly higher value than the Placebo group at 4 weeks. $*$ : $<<0$

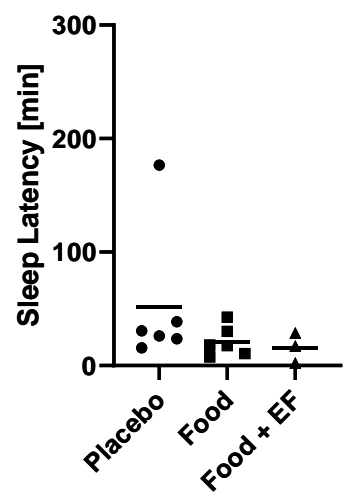

(a) Sleep latency

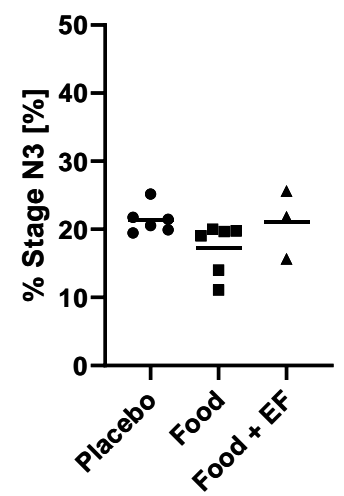

(b) Percentage of sleep N3

Figure 7. (a) Sleep latency and (b) percentage of sleep N3 at week 4. Intergroup differences were analyzed using the Tukey-Kramer test. Sleep latency and the amount of stage N3 showed no statistically significant difference between the three groups. 


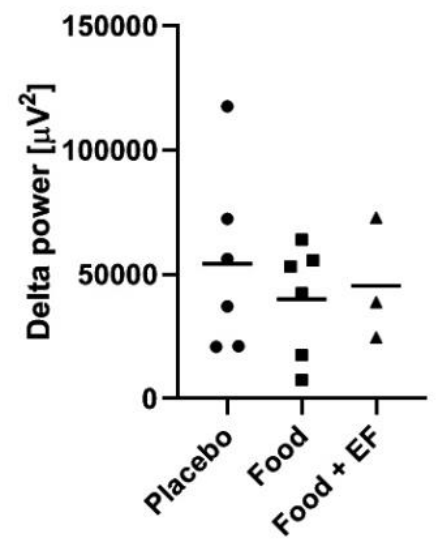

(a) 0 week

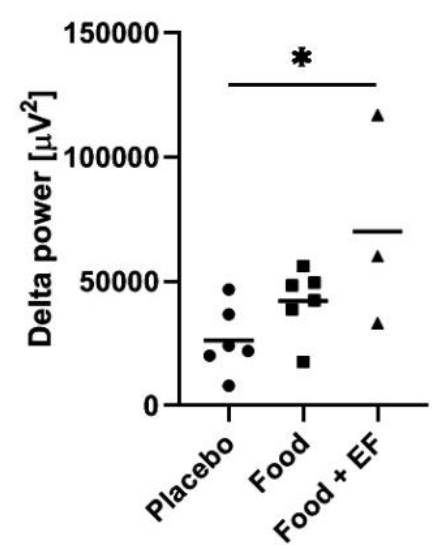

(b) 4 week

Figure 8. Delta power at (a) 0 week and (b) 4 weeks. Inter-group differences were analyzed using Tukey-Kramer test. Food plus EF group showed significantly higher value than that of Placebo group at 4 weeks. ${ }^{*}: p<0.05$

The OSA sleep inventory showed that intragroup changes were significant in Factor II (Initiation and maintenance of sleep) of the Food plus EF group and Factor V (Sleep time) of the Food group (Table 3).

Results of the VAS questionnaire for sleep and fatigue revealed that intragroup changes were significant in "Subjective sleep time" of the Food group and "Waking up" of the Placebo group and Food group (Table 4).
The VAS questionnaire for physical condition was not significantly different between the pre and post intervention in the Food plus EF group (Table 5). Meanwhile, one participant in the Food plus EF group filled in the free comment section, stating that she felt relieved from neck and shoulder stiffness after using the EF therapy device but felt more tired than before.

Table 3. OSA sleep inventory.

\begin{tabular}{|c|c|c|c|c|c|c|c|c|c|c|c|}
\hline \multirow[b]{2}{*}{ Factor I } & \multirow{2}{*}{$\begin{array}{l}\text { Group } \\
\text { Placebo }\end{array}$} & \multirow{2}{*}{$\frac{n}{6}$} & \multicolumn{3}{|c|}{ O week } & \multicolumn{3}{|c|}{4 week } & \multicolumn{3}{|c|}{$\Delta 4$ week } \\
\hline & & & 40.9 & \pm & 4.5 & 44.3 & \pm & 8.9 & 3.3 & \pm & 5.8 \\
\hline \multirow[t]{2}{*}{ Sleepiness on rising } & Food & 6 & 42.8 & \pm & 9.2 & 51.8 & \pm & 12.6 & 9.0 & \pm & 12.5 \\
\hline & Food + EF & 3 & 34.9 & \pm & 5.0 & 43.7 & \pm & 6.6 & 8.8 & \pm & 8.2 \\
\hline Factor II & Placebo & 6 & 34.9 & \pm & 5.9 & 34.4 & \pm & 13.1 & -0.5 & \pm & 9.6 \\
\hline Initiation and & Food & 6 & 32.9 & \pm & 7.6 & 44.9 & \pm & 7.6 & 12.0 & \pm & 14.6 \\
\hline maintenance of sleep & Food $+E F$ & 3 & 26.1 & \pm & 4.3 & 44.9 & \pm & 6.8 & 18.7 & \pm & $4.8^{*}$ \\
\hline Factor III & Placebo & 6 & 43.0 & \pm & 10.3 & 41.8 & \pm & 9.0 & -1.1 & \pm & 14.8 \\
\hline \multirow[t]{2}{*}{ Dream } & Food & 6 & 45.8 & \pm & 17.1 & 39.7 & \pm & 13.4 & -6.1 & \pm & 20.3 \\
\hline & Food $+E F$ & 3 & 38.8 & \pm & 7.3 & 53.9 & \pm & 7.8 & 15.1 & \pm & 14.0 \\
\hline Factor IV & Placebo & 6 & 38.7 & \pm & 7.4 & 42.7 & \pm & 14.0 & 3.9 & \pm & 10.5 \\
\hline \multirow[t]{2}{*}{ Recovery from Fatigue } & Food & 6 & 37.8 & \pm & 10.9 & 54.8 & \pm & 10.3 & 17.0 & \pm & 16.5 \\
\hline & Food $+E F$ & 3 & 33.2 & \pm & 9.1 & 39.9 & \pm & 2.9 & 6.7 & \pm & 12.0 \\
\hline Factor V & Placebo & 6 & 40.0 & \pm & 4.2 & 43.9 & \pm & 9.6 & 3.9 & \pm & 8.6 \\
\hline \multirow[t]{2}{*}{ Sleep time } & Food & 6 & 38.6 & \pm & 4.3 & 50.9 & \pm & 9.1 & 12.4 & \pm & 10.2 * \\
\hline & Food $+E F$ & 3 & 40.0 & \pm & 4.4 & 42.7 & \pm & 8.7 & 2.8 & \pm & 9.6 \\
\hline
\end{tabular}


Table 4. Visual analog scale questionnaire for sleep and fatigue.

\begin{tabular}{|l|l|l|l|l|l|l|l|l|l|l|l|} 
& \multicolumn{1}{|c|}{ Group } & $\mathrm{n}$ & \multicolumn{3}{|c|}{ o week } & \multicolumn{3}{c|}{ 4 week } & \multicolumn{3}{c|}{ 44 week } \\
\hline Subjective & Placebo & 6 & 7.28 & \pm & 1.07 & 6.07 & \pm & 2.47 & -1.22 & \pm & 2.12 \\
\hline sleep time & Food & 6 & 6.85 & \pm & 1.48 & 3.20 & \pm & 2.20 & -3.65 & \pm & $2.33 *$ \\
\hline & Food + EF & 3 & 8.13 & \pm & 0.42 & 4.17 & \pm & 1.89 & -3.97 & \pm & 1.53 \\
\hline Sleep latency & Placebo & 6 & 7.45 & \pm & 1.96 & 7.25 & \pm & 2.38 & -0.20 & \pm & 2.09 \\
\hline & Food & 6 & 7.13 & \pm & 1.25 & 4.33 & \pm & 3.46 & -2.80 & \pm & 3.16 \\
\hline & Food + EF & 3 & 8.00 & \pm & 1.30 & 4.40 & \pm & 2.07 & -3.60 & \pm & 1.97 \\
\hline Waking up & Placebo & 6 & 6.60 & \pm & 2.34 & 3.70 & \pm & 3.20 & -2.90 & \pm & $2.75 *$ \\
\hline & Food & 6 & 6.00 & \pm & 2.52 & 2.65 & \pm & 2.30 & -3.35 & \pm & $1.94 *$ \\
\hline & Food + EF & 3 & 7.90 & \pm & 1.65 & 5.37 & \pm & 1.63 & -2.53 & \pm & 3.24 \\
\hline Physical fatigue & Placebo & 6 & 6.52 & \pm & 2.59 & 5.38 & \pm & 3.37 & -1.13 & \pm & 2.37 \\
\hline & Food & 6 & 6.63 & \pm & 2.28 & 3.15 & \pm & 2.44 & -3.48 & \pm & 3.83 \\
\hline & Food + EF & 3 & 7.37 & \pm & 1.63 & 5.63 & \pm & 1.00 & -1.73 & \pm & 2.48 \\
\hline Mental fatigue & Placebo & 6 & 5.95 & \pm & 2.25 & 4.48 & \pm & 4.01 & -1.47 & \pm & 2.46 \\
\hline & Food & 6 & 6.13 & \pm & 2.22 & 2.13 & \pm & 1.45 & -4.00 & \pm & 3.03 \\
\hline & Food + EF & 3 & 8.00 & \pm & 0.46 & 4.93 & \pm & 1.56 & -3.07 & \pm & 1.93 \\
\hline
\end{tabular}

Intragroup changes were analyzed using Wilcoxon signed rank test. *: $p<0.05$

Table 5. Visual analog scale questionnaire for physical condition.

\begin{tabular}{|c|c|c|c|c|c|c|c|c|c|c|c|}
\hline \multirow[b]{2}{*}{ Pain in the back or waist } & \multirow{2}{*}{$\begin{array}{l}\text { Group } \\
\text { Food + EF }\end{array}$} & \multirow{2}{*}{$\begin{array}{l}n \\
3\end{array}$} & \multicolumn{3}{|c|}{0 week } & \multicolumn{3}{|c|}{4 week } & \multicolumn{3}{|c|}{$\Delta 4$ week } \\
\hline & & & 7.63 & \pm & 1.68 & 5.17 & \pm & 2.40 & -2.47 & \pm & 1.01 \\
\hline Shoulder stiffness & Food $+E F$ & 3 & 9.23 & \pm & 0.12 & 5.43 & \pm & 3.17 & -3.80 & \pm & 3.24 \\
\hline Appetite & Food $+E F$ & 3 & 4.97 & \pm & 1.32 & 2.57 & \pm & 1.88 & -2.40 & \pm & 1.11 \\
\hline Pain in the hands, feet, or joints & Food $+E F$ & 3 & 6.23 & \pm & 3.38 & 4.80 & \pm & 3.65 & -1.43 & \pm & 0.75 \\
\hline Palpitation and breath shortness & Food $+E F$ & 3 & 5.83 & \pm & 1.03 & 3.13 & \pm & 2.17 & -2.70 & \pm & 2.36 \\
\hline Blurred vision or eyestrain & Food $+E F$ & 3 & 8.27 & \pm & 1.72 & 7.63 & \pm & 1.14 & -0.63 & \pm & 1.05 \\
\hline Constipation & Food $+E F$ & 3 & 4.87 & \pm & 3.76 & 3.77 & \pm & 2.80 & -1.10 & \pm & 1.64 \\
\hline Upset stomach or stomachache & Food $+E F$ & 3 & 6.33 & \pm & 1.50 & 2.83 & \pm & 2.69 & -3.50 & \pm & 1.44 \\
\hline Irritation & Food $+E F$ & 3 & 7.40 & \pm & 1.01 & 4.93 & \pm & 2.15 & -2.47 & \pm & 1.72 \\
\hline Blood pressure abnormality & Food $+E F$ & 3 & 3.90 & \pm & 2.77 & 2.10 & \pm & 0.87 & -1.80 & \pm & 3.35 \\
\hline Headache or heaviness of the head & Food + EF & 3 & 7.90 & \pm & 1.82 & 5.23 & \pm & 2.34 & -2.67 & \pm & 3.09 \\
\hline
\end{tabular}

Intragroup changes were analyzed using Wilcoxon signed rank test.

\section{DISCUSSION}

This study investigated the effect of administering functional food, EF therapy device, and both on sleep quality for 4 weeks by measuring the sleep EEG and assessing the subjective symptoms. The intra- and intergroup differences of the three groups were also assessed.
First, SE showed no significant differences in intragroup comparisons nor in intergroup comparisons of pre-post differences (Data not shown). In contrast, SE at week 4 was significantly higher in the Food group and Food plus EF group than in the Placebo group (Fig. 5). This change probably reflected the intervention effects 
because no significant difference was noted at week 0 . In fact, pre-post improvements were significant for "Sleep time" in OSA sleep inventory (Table 3) and

"Subjective sleep time" in VAS questionnaire (Table 4) for the Food group, and "Initiation and maintenance of sleep" in OSA sleep inventory (Table 3) for the Food plus EF group; thus, subjective improvement also occur. Accordingly, SE improved in the Food group and Food plus EF group.

Conversely, sleep latency (Fig. 7-a) and stage N3 (Fig. 7-b) was not significantly different between the groups. Hence, the intervention had an effect to elevate $\mathrm{SE}$, rather than to induce sleep or increase the total amount of deep sleep stage.

Sleep is scored as stage N3 when $20 \%$ or more of an epoch consists of slow wave activity, or delta wave of $0.5 \mathrm{~Hz}$ to $2 \mathrm{~Hz}$ with peak-to-peak amplitude of more than $75 \mu \mathrm{V}$. Thus, the delta wave content can be different in the same stage N3. In fact, the EEG delta power in the first sleep cycle was higher at week 4 in the Food plus EF group than the Placebo group (Fig. 8-b), suggesting that sleep became deeper by the intervention. In addition, SE including sleep latency significantly improved at week 4 in the Food plus EF group as compared to the Placebo group (Fig. 6-b). Taken together, EF treatment exhibited a synergetic effect on sleep quality.

Currently available sleep studies using the EF therapy device were conducted mainly by subjective questionnaire survey, and objective measurements were mostly performed using an actigram and body movements. Sleep structure, such as the discrimination of sleep stage N1 and stage N2, cannot be analyzed without EEG measurement. To our knowledge, our study is the first to measure sleep with EEG for the study of EF therapy device. Additionally, no previous studies have reported improvements in SE.

This study is also the first to evaluate the combined effect of functional food intake and EF therapy device use. The food sample used in this study was composed of ingredients such as lactic-fermented barley GABA (120 mg), lafma extract (50 mg), 0.1\% vitamin B12 (3 $\mathrm{mg})$, vitamin B6 (1.5 mg). GABA reportedly has an effect on sleep quality. In a previous review, benefits of GABA consumption at the early sleep stage could be associated with GABA's stress reduction properties rather than direct sleep-inducing and/or -maintaining benefits per se [13]. Lafma (Apocynum venetum) has anti-platelet aggregation [27], antioxidant [28-29], antihypertensive, antihyperlipidemic, antidepressant, and antidiabetic effects [30] because of its various flavonoid components. The combination of lafma and GABA improves sleep quality in a human clinical trial [31]. Moreover, vitamin B12 deficiency has been linked to depression and insomnia. And then, the combination of vitamin B complex, melatonin, and magnesium effectively improved sleep quality in 60 people diagnosed with insomnia [15]. Sleep-promoting effect of these ingredients was observed in our study.

Physical examination revealed the systolic blood pressure was significantly lowered in the Placebo group. This can be attributed to the higher initial value for the Placebo group than that for the other groups.

We have developed a functional food using Kumasasa (Sasa senanensis) leaf, which contains amino acids, vitamin $\mathrm{K}$, polysaccharides, and low-molecule compounds, such as p-coumaric acid, ferulic acid, vanillin, apigenin, luteolin, tricin, syringaresinol, phenylpropanoid, $p$-hydroxybenzoic acid, p-hydroxybenzaldehyde, and 3,4-dihydroxybenzaldehyde [18-19]. Kumasasa demonstrated antiultraviolet [20], antiviral [21], and antioxidation effects in a rat mesenchymal ischemia-reperfusion model [22], an antitumor effect in mice K562 cells (human chronic myeloid leukemia) and YAC1 cells (murine lymphoma) [23], and an improvement effect for oral environments [24]. It can also inhibit nervous injury by amyloid beta 
[25]; nitric oxide and prostaglandin E2 from macrophages; and histamine release from rat peritoneal cells [26]. Although relaxation and sleep-inducing effects of Kumasasa are not reported, antioxidation and immune modulation effects are expected. Thus, Kumasasa powder was used as an additional ingredient.

According to clinical studies of the EF therapy device, EF enhances EEG theta wave [8] and sleep quality [7]. In an animal study, EF suppressed stress hormone [5-6] and inflammatory compounds [5]. Similar to GABA intake, EF may elicit sleep-improving effect via stress reduction.

Although an ELF-EF is higher on the body surface, it is significantly attenuated inside the human body because of its shielding effect. Internal EFs were in the order of magnitude of $\mathrm{mV} / \mathrm{m}$

[8]. The insight into whether the internal EFs elicit the physiological response, or the body surface EF is transduced to the physiological system remains uncertain.

Additionally, the food samples used in this experiment were formulated with ingredients that have already been reported for their effects on sleep [32]. This study hopes that the combined use of the healthy food formula and the EF therapy device will improve the sleep-related problems of modern people.

Limitation of this study is that the number of participants was small, especially in the Food plus EF group. In addition, solitary effect of EF application remains unknown due to combination with the functional food. Further research with more participants and with "EF only group" is needed.

\section{CONCLUSIONS}

In conclusion, sleep efficiency is improved by consuming food containing sleep-related ingredients such as GABA and lafma and is enhanced when the administration of such food formula is combined with EF application.
List of Abbreviations: EEG: electroencephalography, EF: electric field, GABA: gamma-aminobutyric acid, OSA: Oguri-Shirakawa-Azumi, REM: Rapid eye movement, SE: sleep efficiency, SL: sleep latency, SPT: Sleep period time, TST: Total sleep time, VAS: visual analog scale.

Competing Interests: The human trial was supported by funding from Hakuju Institute for Health Science Co., Ltd. and was outsourced to Oneness Support Inc. Although T.N., K. H. and S. H. are employees of Hakuju Institute for Health Science Co., Ltd., these authors are not involved in the interpretation of the results of human experiments, and the independence of the investigators and the study is ensured. The other authors have no conflict of interests to declare.

Authors' Contributions: T.N., K. H. and S. H. designed the research protocol. K. H. provided test and placebo foods tablet. N.M. is investigator of this study and analyzed the physical data for evaluation of adverse events. K.W. performed statistical analysis. T.N., S.H. and K.W. wrote the manuscript. T.N. and K.W. reviewed and edited the manuscript. K.W. had primary responsibility for the final content. All authors read and approved the final version of the manuscript.

Acknowledgements: We would like to thank Makoto Terashima and Hitoe Ushirodani of Oneness Support Inc. for their cooperation and advice in the human trial. We would also like to thank Enago (www.enago.jp) for the English language review.

\section{REFERENCES}

1. Tao S, Wu X, Zhang Y, Zhang S, Tong S, Tao F: Effects of sleep quality on the association between problematic mobile phone use and mental health symptoms in Chinese college students. Int J Environ Res Public Health 2017, 14:E185. https://doi.org/10.3390/ijerph14020185

2. Suzuki M, Taniguchi T, Furihata R, Yoshita K, Arai Y, Yoshiike N, Uchiyama M: Seasonal changes in sleep duration and sleep problems: a prospective study in Japanese community residents. 
Functional Foods in Health and Disease 2021; 11(12): 659-672

PLoS One 2019, 14:e0215345.

\section{https://doi.org/10.1371/journal.pone.0215345}

3. Yamamoto K, Motokawa K, Yoshizaki T, Yano T, Hirano H, Ohara Y, Shirobe M, Inagaki H, Awata S, Shinkai S, Watanabe Y: Association of dietary variety and appetite with sleep quality in urban-dwelling older Japanese adults. The Journal of Nutrition, Health and Aging 2020, 24:152-159.

https://doi.org/10.1007/s12603-019-1297-4

4. Katagiri R, Asakura K, Kobayashi S, Suga H, Sasaki S: Low intake of vegetables, high intake of confectionary, and unhealthy eating habits are associated with poor sleep quality among middle-aged female Japanese workers. J Occup Health 2014, 56:359-368. https://doi.org/doi.org/10.1539/joh.14-0051-OA

5. Harakawa S, Nedachi T, Suzuki H: Extremely low-frequency electric field suppresses not only induced stress response but also stress-related tissue damage in mice. Sci Rep 2020, 10:20930. https://doi.org/10.1038/s41598-020-76106-1

6. Hori T, Nedachi T, Suzuki H, Harakawa S: Characterization of the suppressive effects of extremely-low-frequency electric fields on a stress-induced increase in the plasma glucocorticoid level in mice. Bioelectromagnetics 2018, 39:516-528.

https://doi.org/10.1002/bem.22138

7. Ohtsuki T, Nabeta T, Nakanishi H, Kawahata H, Ogihara T, Morishita R, Aoki M: Electric field exposure improves subjective symptoms related to sleeplessness in college students: a pilot study of electric field therapy for sleep disorder. Immunology, Endocrine and Metabolic Agents in Medicinal Chemistry 2017, 17:37-48. https://doi.org/10.2174/1871522217666170815163329

8. Shinba T, Nedachi T, Harakawa S: Extremely low-frequency electric field exposure increases theta power of EEG in both eyes-open and eyes-closed resting conditions in healthy male subjects. IEEJ Transactions on Electrical and Electronic Engineering 2021, 16:592-599.

https://doi.org/10.1002/tee.23334

9. Bannai M, Kawai N: New therapeutic strategy for amino acid medicine: glycine improves the quality of sleep. J Pharmacol Sci 2012, 118:145-148. https://doi: 10.1254/jphs.11r04fm

10. Ito $Y$, Takahashi S, Shen M, Yamaguchi K, Satoh M: Effects of L-serine ingestion on human sleep. Springerplus 2014, 3:456. https://doi.org/10.1186/2193-1801-3-456

11. Kim S, Jo K, Hong K-B, Han SH, Suh HJ: GABA and L-theanine mixture decreases sleep latency and improves NREM sleep. Pharmaceutical Biology 2019, 57:64-72.

https://doi.org/10.1080/13880209.2018.1557698

12. Miyake M, Kirisako T, Kokubo T, Miura Y, Morishita K, Okamura H, $\underline{\text { FFHD }}$

Page 671 of 672

Tsuda A: Randomised controlled trial of the effects of L-ornithine on stress markers and sleep quality in healthy workers. Nutr J 2014, 13:53.

https://doi.org/10.1186/1475-2891-13-53

13. Hepsomali P, Groeger JA, Nishihira J, Scholey A: Effects of Oral Gamma-Aminobutyric Acid (GABA) Administration on Stress and Sleep in Humans: A Systematic Review. Front Neurosci 2020, 14:923. https://doi: 10.3389/fnins.2020.00923

14. Miyakawa M, Kubo S, Oda H, Motoki N, Mizuki M, Tsukahara T, Tanaka M, Yamauchi K, Abe F, Nomiyama T: Effects of lactoferrin on sleep conditions in children aged 12-32 months: a preliminary, randomized, double-blind, placebo-controlled trial. Nat Sci Sleep 2020, 12:671-677. https://doi.org/10.2147/NSS.S263106

15. Djokic G, Vojvodić P, Korcok D, Agic A, Rankovic A, Djordjevic V, Vojvodic A, Vlaskovic-Jovicevic T, Peric-Hajzler Z, Matovic D, Vojvodic J, Sijan G, Wollina U, Tirant M, Thuong NV, Fioranelli M, Lotti T: The effects of magnesium - melatonin - vit B complex supplementation in treatment of insomnia. Open Access Maced J Med Sci 2019, 7:3101-3105.

https://doi.org/10.3889/oamjms.2019.771

16. Ito T, Goto K, Takanari J, Miura T, Wakame K, Nishioka H, Tanaka A, Nishihira J: Effects of enzyme-treated asparagus extract on heat shock protein 70 , stress indices, and sleep in healthy adult men. J Nutr Sci Vitaminol (Tokyo) 2014, 60:283-290. https://doi.org/10.3177/jnsv.60.283

17. Xie $\mathrm{W}$, Zhang $\mathrm{X}$, Wang $\mathrm{T}$, Hu J: Botany, traditional uses, phytochemistry and pharmacology of Apocynum venetum $\mathrm{L}$. (luobuma): a review. J Ethnopharmacol 2012, 141:1-8. https://doi.org/10.1016/j.jep.2012.02.003

18. Sato K, Tatsunami R, Nakata A, Komatsu KI, Harakawa S, Nedachi T, Haketa K, Inagawa H, Wakame K: Effects of Kumaizasa (Sasa senanensis) leaf extract on innate immune regulation in HEK293 cells and macrophages. Anticancer Res 2021, 41:4093-4100. https://doi.org/10.21873/anticanres.15212

19. Matsuta T, Sakagami H, Satoh K, Kanamoto T, Terakubo S, Nakashima H, Kitajima M, Oizumi H, Oizumi T: Biological activity of luteolin glycosides and tricin from Sasa senanensis Rehder. In Vivo 2011, 25:757-762.

20. Matsuta T, Sakagami H, Sugiura T, Kitajima M, Oizumi H, Oizumi T: Structural characterization of anti-UV components from Sasa senanensis Rehder extract. In Vivo 2013, 27:77-83.

21. Sakagami H, Fukuchi $K$, Kanamoto T, Terakubo $S$, Nakashima $H$, Natori T, Suguro-Kitajima M, Oizumi H, Yasui T, Oizumi T: Synergism of alkaline extract of the leaves of Sasa senanensis Rehder and antiviral agents. In Vivo 2016, 30:421-426. 
22. Kurokawa T, Itagaki S, Yamaji T, Nakata C, Noda T, Hirano T, Iseki K: Antioxidant activity of a novel extract from bamboo grass (AHSS) against ischemia-reperfusion injury in rat small intestine. Biol Pharm Bull 2006, 29:2301-2303.

https://doi.org/10.1248/bpb.29.2301

23. Seki $\mathrm{T}$, Kida $\mathrm{K}$, Maeda $\mathrm{H}$ : Immunostimulation-mediated anti-tumor activity of bamboo (Sasa senanensis) leaf extracts obtained under 'vigorous' condition. Evid Based Complement Alternat Med 2010, 7:447-457.

https://doi.org/10.1093/ecam/nen026

24. Sakagami $H$, Sheng $H$, Ono $K$, Komine $Y$, Miyadai T, Terada $Y$, Nakada D, Tanaka S, Matsumoto M, Yasui T, Watanabe K, Junye J, Natori T, Suguro-Kitajima M, Oizumi H, Oizumi T: Anti-halitosis effect of toothpaste supplemented with alkaline extract of the leaves of Sasa senanensis Rehder. In Vivo 2016, 30:107-111.

25. Sakagami $H$, Tsuji $M$, Tomomura $M$, Masuda $Y$, Iwama $S$, Nakagawa M, Suzuki H, Tanaka K, Abe T, Tamura N, Tomomura A, Yokose S, Takeshima H, Natori T, Horiuchi M, Fujisawa T, Kiuchi Y, Oguchi K, Yasui T, Oizumi H, Oizumi T: Protection of differentiating neuronal cells from amyloid $\beta$ peptide-induced injury by alkaline extract of leaves of Sasa senanensis Rehder. In Vivo 2018, 32:231-239.

26. Zhou L, Hashimoto K, Satoh K, Yokote Y, Kitajima M, Oizumi T, Oizumi H, Sakagami H: Effect of Sasa senanensis Rehder extract on NO and PGE2 production by activated mouse macrophage-like RAW264.7 cells. In Vivo 2009, 23:773-777.
27. Kasimu R, Fan Z, Wang X, Hu J, Wang P, Wang J: Anti-platelet aggregation activities of different fractions in leaves of Apocynum venetum L. J Ethnopharmacol 2015, 168:116-121. https://doi.org/10.1016/j.jep.2015.03.013

28. Feng Y, Jiang C, Yang F, Chen Z, Li Z: Apocynum venetum leaf extract protects against $\mathrm{H}_{2} \mathrm{O}_{2}$-induced oxidative stress by increasing autophagy in PC12 cells. Biomed Rep 2020, 13:6. https://doi.org/10.3892/br.2020.1313

29. Liang T, Yue W, Li Q: Comparison of the phenolic content and antioxidant activities of Apocynum venetum L. (luo-bu-ma) and two of its alternative species. Int J Mol Sci 2010, 11:4452-4464. https://doi.org/10.3390/ijms11114452

30. Shen J, Yang K, Jiang C, Ma XQ, Zheng MX, Sun CH: Development and application of a rapid HPLC method for simultaneous determination of hyperoside, isoquercitrin and eleutheroside $E$ in Apocynum venetum L. and Eleutherococcus senticosus. BMC Chem 2020, 14:35 https://doi.org/10.1186/s13065-020-00687-1

31. Yamatsu A, Yamashita Y, Maru I, Yang J, Tatsuzaki J, Kim M: The improvement of sleep by oral intake of GABA and Apocynum venetum leaf extract. J Nutr Sci Vitaminol (Tokyo) 2015, 61:182-187. https://doi.org/10.3177/jnsv.61.182

32. Zeng Y, Yang J, Du J, Pu X, Yang X, Yang S, Yang T: Strategies of functional foods promote sleep in human being. Curr Signal Transduct Ther 2014, 9:148-155.

https://doi.org/10.2174/1574362410666150205165504 